\title{
Few-mode fiber coupled superconducting nanowire single-photon detectors for photon efficient optical communications
}

\author{
Brian E. Vyhnalek, Sarah A. Tedder, Evan J. Katz, and Jennifer M. Nappier \\ National Aeronautics and Space Administration \\ Glenn Research Center \\ Cleveland, OH, USA
}

\begin{abstract}
The NASA Glenn Research Center's development of a high-photon efficiency real-time optical communications ground receiver has added superconducting nanowire single-photon detectors (SNSPDs) coupled with few-mode fibers (FMF). High data rate space-to-ground optical communication links require enhanced ground receiver sensitivity to reduce spacecraft transmitter constraints, and therefore require highly efficient coupling from fiber to detector. In the presence of atmospheric turbulence the received optical wavefront can be severely distorted introducing higher-order spatial mode components to the received signal. To reduce mode filtering and mismatch loss and the resulting degradations to detector coupling efficiency, we explore the use of few-mode fiber coupling to commercial single-pixel SNSPDs. Graded index 20- $\mu \mathrm{m}$ few-mode fibers allow the commercial single pixel SNSPD's active area to couple with equal efficiency as single mode fibers. Here we determine detector characteristics such as count rate, detection efficiency, dark counts, and jitter, as well as detection efficiencies for higher-order fiber spatial modes. Additionally, we assess the laboratory performance of the detectors in an optical system which emulates future deep space optical communications links.
\end{abstract}

Keywords: Optical communications, single photon detectors, superconducting nanowire, few-mode fibers

\section{INTRODUCTION}

As data return demands from science missions continue to increase, future NASA communications systems will require the high data return benefit of optical communications. By increasing ground receiver efficiency through improved telescope coupling, advanced light detection capabilities, and improved system design, the overall receiver sensitivity can be enhanced and thereby reduce spacecraft transmitter size, mass and power. A key enabling technology for advanced light detection are supercoducting nanowire single-photon detectors (SNSPDs), which have been demonstrated to offer superior performance in detection effciency, timing resolution, and count rates over semiconductor photodetectors, and have been demonstrated in space-to-ground optical communications links. ${ }^{1}$ With several commercial vendors offering turnkey multichannel SNSPD systems with simplified cryogenics, SNSPDs are increasingly viable as an operational component for space-to-ground highphoton efficiency free-space optical communications links.

To meet the goals of future optical communications systems NASA is developing a real time optical receiver system, which includes the telescope aft optics, single photon counting detectors, and a real time field programmable gate array (FPGA) based receiver. In this paper we report results from recent improvements in our commercial SNSPDs for increased count rates and improved jitter performance. Additionally we have coupled SNSPDs to 20- $\mu$ m core few-mode fibers (FMF), and we show the mode-dependent detection efficiency. Due to atmospheric turbulence, energy in the transmitted beam is transferred from the fundamental Gaussian mode into higher-order spatial mode components. Thus detectors sized to couple to single-mode fiber suffer from modal filtering loss, and hence the receiver has an overall degraded detection efficiency. Larger area SNSPDs are in development, but suffer from lower count rates, higher dark counts, higher jitter, and low yield. Other

Send correspondence to brian.e.vyhnalek@nasa.gov 
options include interleaved SNSPD on-chip arrays which can cover larger ares, ${ }^{2}$ but this technology is mainly in development and only beginning to emerge commercially. Few-mode fibers, with appropriate refractive index grading, can couple equally to SNSPDs as single-mode fiber. Therefore there is no efficiency loss, but increased signal detection capability.

\section{DETECTOR SYSTEM DESCRIPTION AND CHARACTERIZATION}

Previously we characterized a commercial two-channel, single-mode fiber-coupled superconducting nanowire single photon detector system from Quantum Opus, LLC (Figure 1a), assessing detection efficiency at an operating wavelength of $1550 \mathrm{~nm}$, in addition to count rate, timing jitter and system performance in an SCPPM communications link. ${ }^{3}$ The base system consists of a closed-cycle helium cryocooler with a $25 \mathrm{~K}$ first stage and $2.5 \mathrm{~K}$ second stage on which the SNSPDs are mounted, enclosed in a rack-mountabe box. To the two-channel system we have added two addtional SNSPD channels coupled with $20-\mu \mathrm{m}$ core, gradient index few-mode fibers. Efficient coupling from the detectors to single-mode and few-mode optical fiber is achieved through fiber self-alignment, ${ }^{4,5}$ and the fibers are routed through cryogenic feed-thrus to the inputs on the front of the enclosure.

The devices are current biased with adjustable front panel controls, and coupled to $50 \Omega$ coaxial readout cables to room temperature amplifiers with $500 \mathrm{MHz}$ bandwidth and a maximum $55.6 \mathrm{~dB}$ gain. ${ }^{6}$ For additional improvement over the base system, we have had the biasing and readout electronics reconfigured to further optimize the SNSPD electro-thermal feedback for increased count rates and reduced jitter. The system is powered via a Standford Research Systems SIM900 mainframe, and can be computer controlled either serially or through GPIB interface.

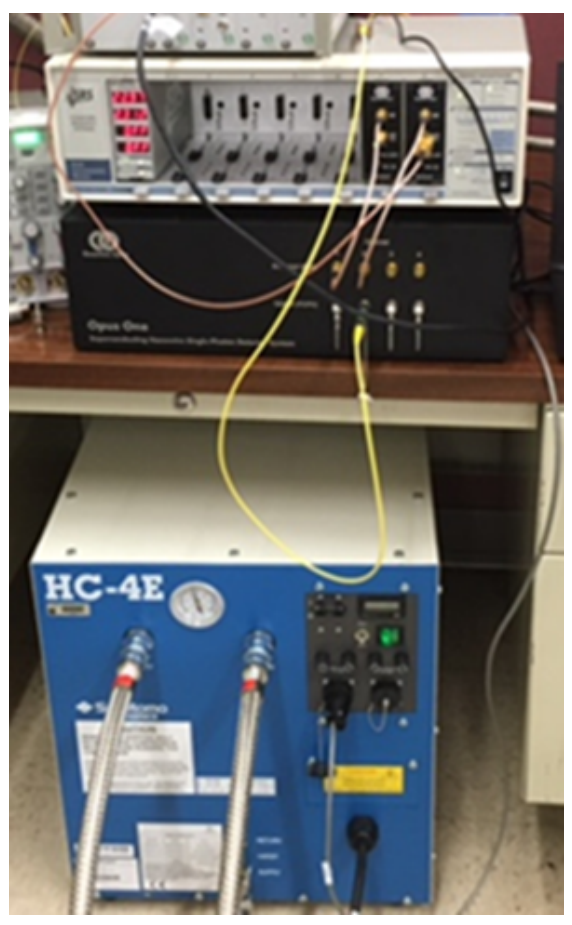

(a)

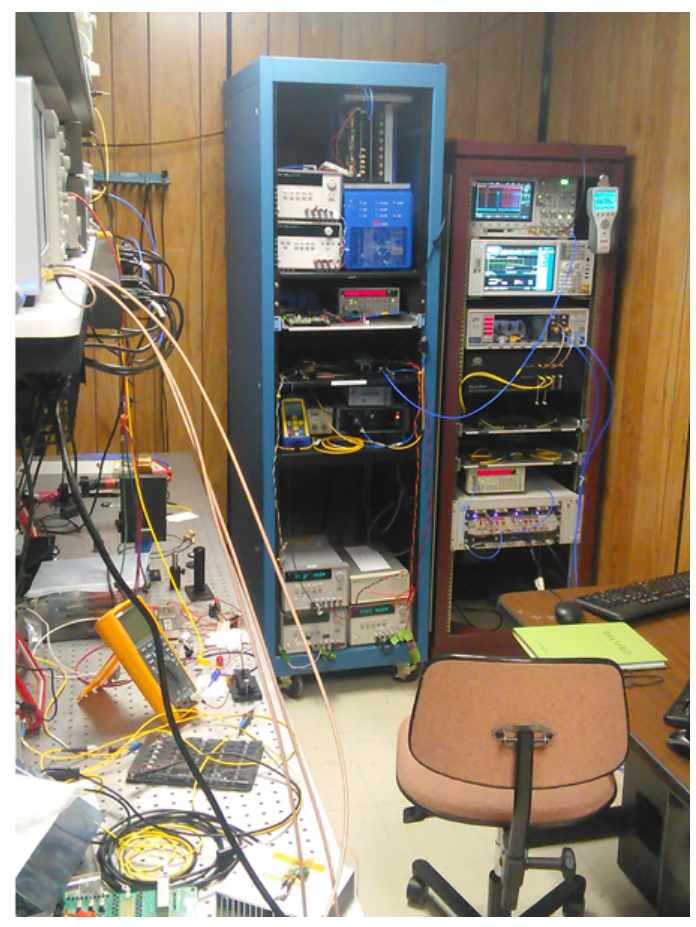

(b)

Figure 1: (a) Opus One ${ }^{T M}$ SNSPD system. (b) SNSPD system rack-mounted. The helium compressor is located in the adjacent room with the flex lines fed through the wall.

We characterized the system detection efficiency (SDE) and detector count rate limitations using the setup shown in Figure 2. The illumination source was a distributed feedback (DFB) laser thermally controlled for a peak wavelength of $1550 \mathrm{~nm}$, and operated with continuous wave $(\mathrm{CW})$ output. The laser output power was 
attenuated with a fixed attenuator to set an upper limit to the input photon flux, and then reduced further up to $60 \mathrm{~dB}$ using an electronically controlled variable attenuator. Following the attenuators a 99/1 splitter was used with the $99 \%$ split to an InGaAs power meter with a $10 \mathrm{pW}$ minimum detection limit. The $1 \%$ split was then split 50/50, with each split sent to fiber paddle polarization controllers before input into the detector enclosure. Output electrical pulses from the SNSPDs were either observed and sampled on a 20 Gsps oscilloscope, or sent to a multichannel time-correlated single photon counting (TCSPC) unit to measure count rates. Finally a computer recorded simultaneous measurements from the TCSPC and optical power meter, in addition to controlling the variable attenuator and SNSPD bias current.

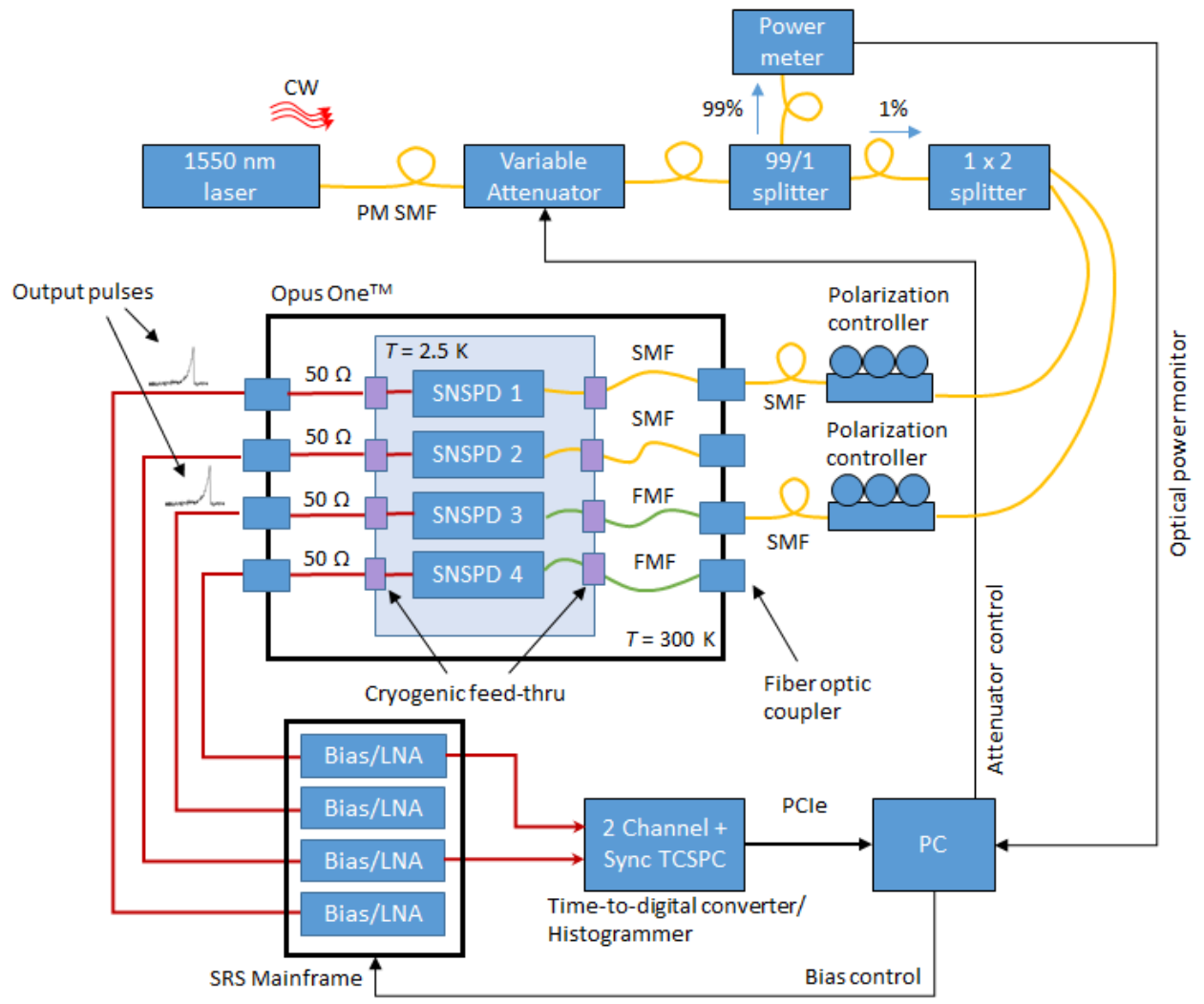

Figure 2: Characterization setup diagram

System detection efficiency was estimated for each detector channel while sweeping over bias current levels at a fixed input photon flux. Initially, background count rates (BCR) were obtained, as shown in Figure 3, with the source laser off and the detector enclosure input ports closed. Note that inside the enclosure there is approximately 1 meter length of fiber at room temperature before being fed through to the cryostat. The data shown is an average of 10 measurements at each bias current, with the bias current normalized by each SNSPD's switching current, $I_{S W}$. For the SMF-coupled detectors (Ch 1 and $\mathrm{Ch} 2$ ), the background counts increased to $\approx$ $1000 \mathrm{cps}$ at $I_{B} \approx 0.97 I_{S W}$ before being dominated by detector dark counts. Also shown in Figure 3 are the SMFcoupled background counts obtained previously, ${ }^{3}$ in which a long-wavelength blackbody mandrel mode filter was in place on the $25 \mathrm{~K}$ first stage of the cryostat. The filter was removed for this set of characterizations due to the potential for spatial mode rejection in the few-mode fibers. As can be seen, with the blackbody filter in place, up to $\approx 10$ or $12 \mathrm{~dB}$ of background count improvement can be achieved. The FMF coupled detectors (Ch 3 and Ch 4) show nearly $20 \mathrm{~dB}$ greater background counts over the SMFs at $I_{B} \approx 0.97 I_{S W}$, with a maximum $B C R$ on the order of $10-100 \mathrm{kcps}$. In accordance with the previous SMF data, it is anticipated that with appropriate filtering the blackbody generated background counts can be reduced by at least one order of magnitude.

The SNSPDs have a polarization dependency, therefore we adjusted the fiber polarization controllers at the 


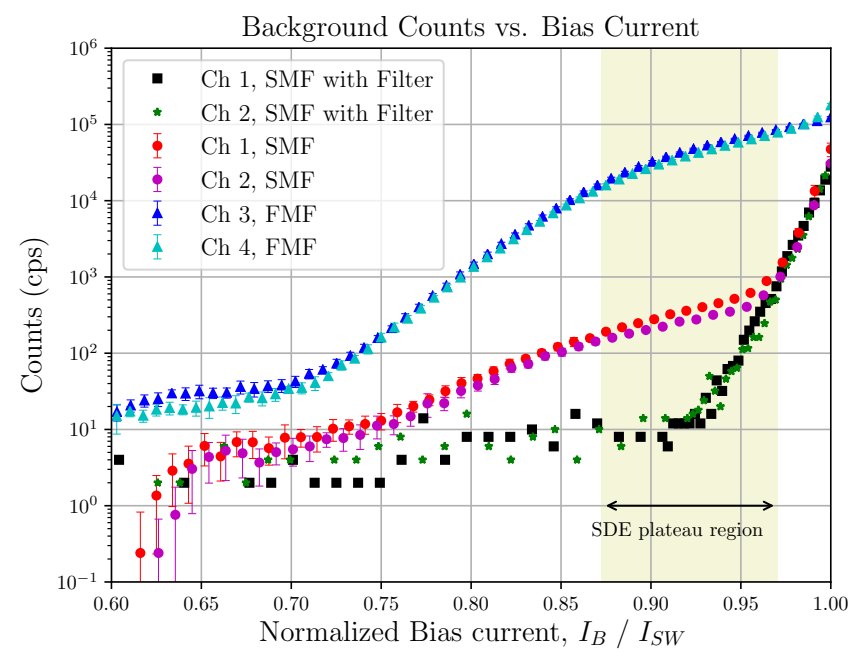

Figure 3: Background count rate vs. bias current.

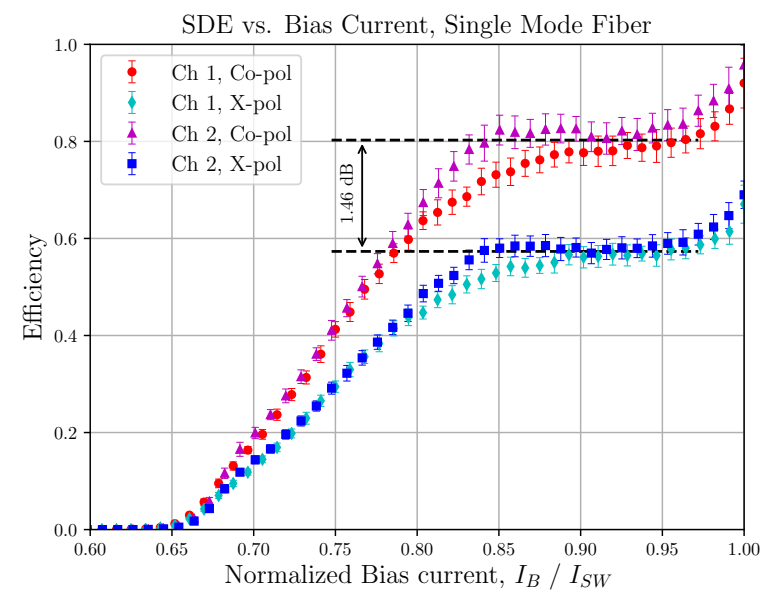

(a)

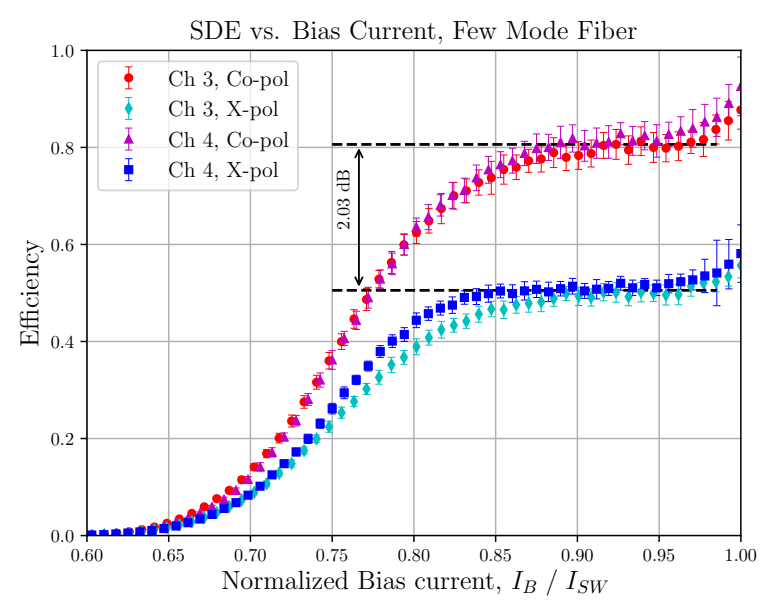

(b)

Figure 4: System detection efficiency (SDE) vs. bias current for (a) single-mode fiber coupled detectors and (b) few-mode fiber coupled detectors.

enclosure inputs to determine the SDE corresponding to the optimal (co-pol) and worst (x-pol) input states of polarization. Figures $4 \mathrm{a}$ and $4 \mathrm{~b}$ show the average and standard deviation of 10 measurements at each bias current, for both the SMF-coupled and FMF-coupled channels. The system detection efficiency was determined by $S D E=\left(R_{\text {out }}-B C R\right) / R_{\text {in }}$, where $R_{\text {out }}$ is the measured output count rate, $B C R$ is the background count rate, and $R_{i n}$ is the estimated input photon flux, in this case attenuated to a constant level of $\approx 500,000 \mathrm{ph} / \mathrm{s}$. We estimated the input photon flux from simultaneous power meter measurements, and through calibration of system losses after the meter to the enclosure input ports. The SNSPDs for all channels shown in Figures 4a and $4 \mathrm{~b}$ display linear detection efficiency increase over the $I_{B} \approx 0.65 I_{S W}-0.85 I_{S W}$ range, after which displaying efficiency plateauing over the $I_{B} \approx 0.85 I_{S W}-0.95 I_{S W}$ bias current range. For both the SMF and FMF channels the SDE for optimal input polarization (co-pol) reached an average plateau value, $\mathrm{SDE}_{\max }$ of $\approx 80-81 \%$. At the worst input state of polarization ( $\mathrm{x}-\mathrm{pol})$, an average plateau value, $\left(\mathrm{SDE}_{\min }\right)$ of $\approx 57 \%$ was obtained for the SMF-coupled detectors and $\approx 51 \%$ for the FMF-coupled detectors. The polarization dependence ratio, 
$r_{\text {pol }}=S D E_{\max } / S D E_{\text {min }}$, was determined to be about factor of $\approx 1.46 \mathrm{~dB}$ on the SMF side and $\approx 2.03 \mathrm{~dB}$ on the FMF side.

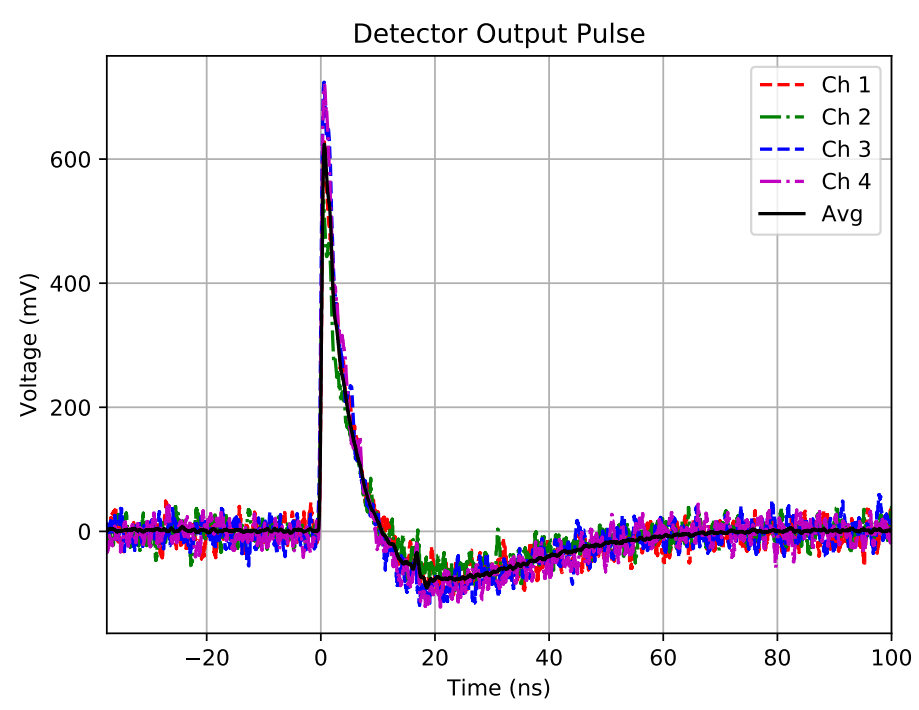

Figure 5: Typical detector output pulses at optimal bias points.

Next, we considered timing characteristics and detector response at a fixed bias point for varying photon flux. Figure 5 , shows typical output pulses for each of the detector channels at a bias levels $I_{B} \approx 0.9 I_{S W}$. The average pulse height for all channels is about $600 \mathrm{mV}$, but depending on bias point can range from $\approx 300 \mathrm{mV}$ - $600 \mathrm{mV}$. Rise time is $\approx 850 \mathrm{ps}$ on average, and the $90 / 10$ fall time of the pulse is around $20-25 \mathrm{~ns}$. Based on this detector dead time, we can infer a maximum count rate of 40 - $50 \mathrm{Mcps}$. However, the true maximum count rate is higher because the detectors are not required to fully reset before subsequent detection events. As a better indication of count rate capability we measured the output counts under variable levels of input intensity, as shown in Figure 6. In this case the detectors were set at a fixed bias for maximum efficiency, and the input optical power was controlled through variable attenuation. As before, the input photon flux was estimated from simultaneous power meter measurements while accounting for system losses and background counts. From Figure 6 it can be seen that the output counts follow a log-linear response up to input photon levels of about 65 - $68 \mathrm{M}$-photons/s, before tending to apparent saturation at an output count rate of $\approx 60-63 \mathrm{Mcps}$. Even so, the output count rate then again continues to increase as relaxation oscillation in the SNSPDs sets in, reaching a absolute maximum approaching $\approx 80-100$ Mcps before complete detector latching at input flux rates on the order of 10 s of G-photons/s.

From the measured output count rates we can determine an effective efficiency as a function of photon flux. In Figure $7 \mathrm{a}$ we show the effective detection efficiency at different intensity input for both optimal polarization (co-pol) and anti-optimal polarization (x-pol). Addtionally, to this data we fit a dead-time model for output counts of the form, ${ }^{7}$

$$
n_{\text {out }}(N)=\frac{p N t}{p-1+\exp (p N \tau)}
$$

where $p \in[0,1]$ is a fitting parameter, $N$ is the input photon count rate, $t$ is the sampling interval, and $\tau$ is the detector dead time, from which we can derive an effective efficiency

$$
\eta_{\text {eff }}(N)=\eta_{\max } \frac{n_{\text {out }}(N)}{N},
$$

where $\eta_{\max }$ is the maximum detection efficiency, for either best or worst polarization. This is an effective efficiency because for a given bias point the true detection efficiency is $\eta_{\max }$, but due to the detector finite 


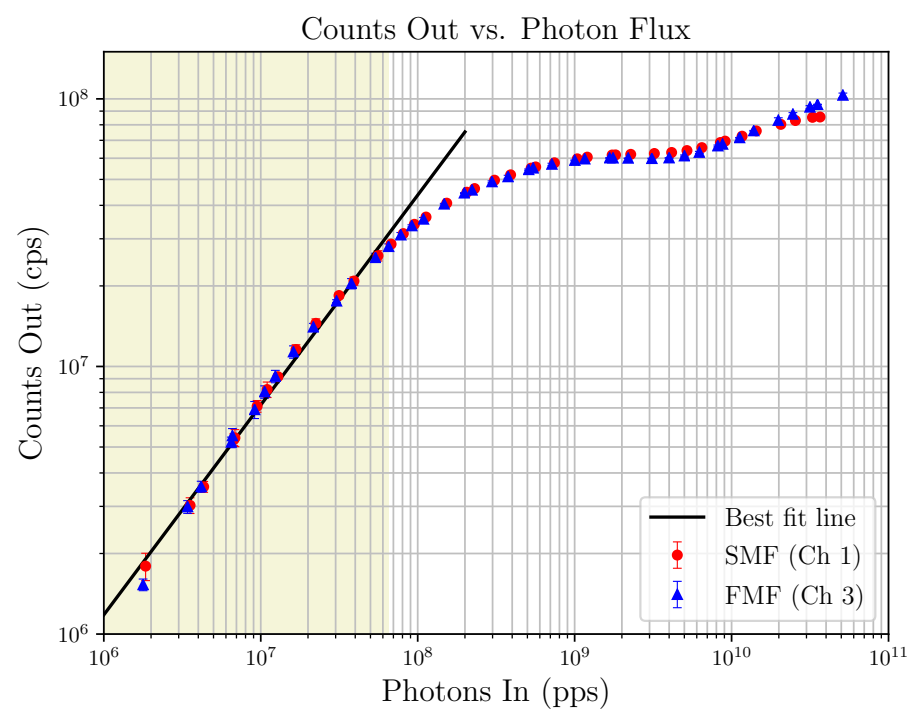

Figure 6: Output counts for variable input photon flux, at optimal polarization. Highlighted is the approximate linear response region.

reset time the detector is partially-to fully blocked depending on the input rate. Figure $7 \mathrm{~b}$ shows the detector blocking loss, i.e. reduction from maximum efficiency, up to $3 \mathrm{~dB}$, which can be a more useful parameter for communication link considerations.

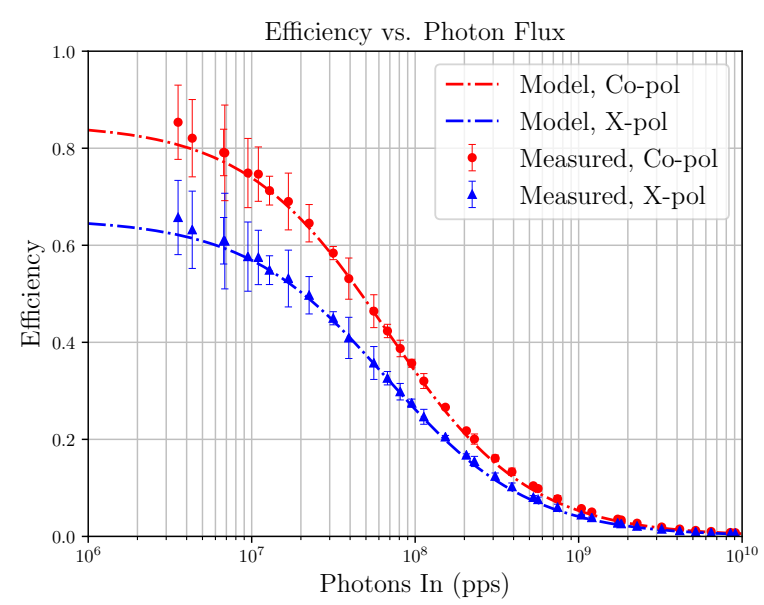

(a)

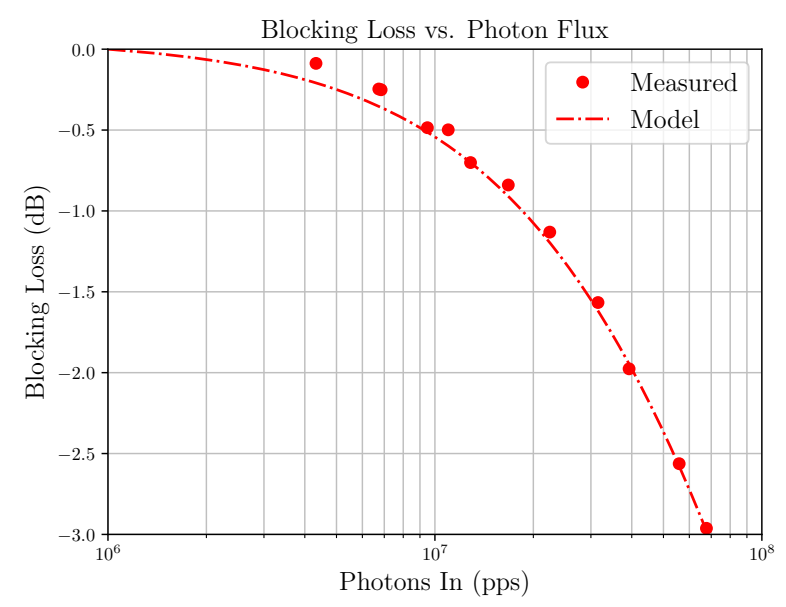

(b)

Figure 7: (a) Effective detection efficiency vs. input photon flux for both co and cross-polarization. (b) Detector blocking loss vs. photon flux.

Next we characterized the system instrument response function (IRF), and derived the detection jitter as a function of bias current using the modified setup shown in Figure 8. A femtosecond fiber laser with $1550 \mathrm{~nm}$ peak wavelength was used in place of the CW DFB laser. This laser output pulses with a minimum of $100 \mathrm{fs}$ FWHM at a $50 \mathrm{MHz}$ repetition rate, and was attenuated to a level of $\ll 1$ photon/pulse on average, giving an effective pulse repetition rate of $1 \mathrm{MHz}$. An electrical pulse was also simultaneously output from the laser synchronized 
with the rising edge of the optical pulse, and was fed into the synchronization input of the TCSPC card. The TCSPC card then measured the time interval between the rising edge of the electrical sync pulse from the laser and the rising edge of the output pulse from the SNSPDs, and built up statistical distributions with 13 ps bins.

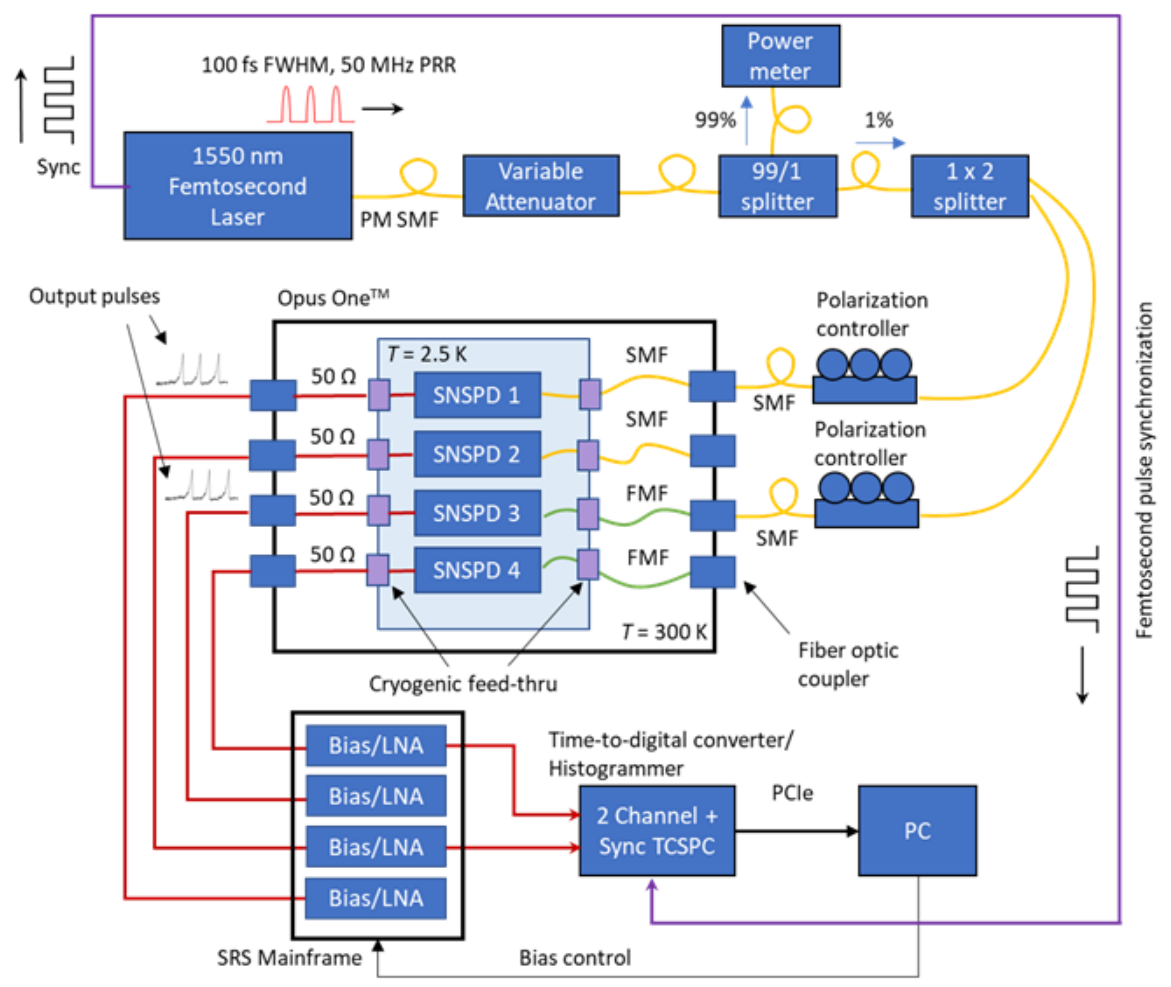

Figure 8: IRF/Detector jitter setup diagram

For each of the measured IRFs, for example as shown in Figure 9, we fit a Gaussian distribution. Then from the fitted distributions we extracted the total measurement jitter, defined as the FWHM of the IRF. ${ }^{8}$ From Figure 9 it can be seen that the width of the IRF decreases with increasing $I_{B}$, consistent with the fact that at higher $I_{B}$ the output pulse amplitudes are larger, and therefore higher signal-to-noise ratio. ${ }^{9}$ However, these values also include jitter effects from the input laser, input sync pulse, and TCSPC card, thus the system jitter $J_{S}$ can be determined from ${ }^{8}$

$$
J_{\text {meas }}^{2}=J_{\text {laser }}^{2}+J_{\text {sync }}^{2}+J_{T C S P C}^{2}+J_{S}^{2} .
$$

With $J_{\text {laser }}=0.06 \mathrm{ps}, J_{\text {sync }}=4.0 \mathrm{ps}$, and $J_{T C S P C}=20.0 \mathrm{ps}, J_{S}$ was calculated for each channel, and the dependence on bias current $I_{B}$ within the maximum efficiency operating region is shown in Figure 10. Here again it can be seen that the jitter decreases linearly with increasing bias current. Channels 3 and 4 show the best dependence, with measured values decreasing over the range from $<\approx 80$ ps to $<50$ ps. The measured Channel 1 jitter decreases similarly, but with higher overall jitter levels offset by $10-20$ ps. Channel 2 shows the highest overall detection jitter, but also with the most $I_{B}$ dependence, attaining $<100$ ps for $I_{B} \approx 0.92 I_{S W}$, but decreasing to $<\approx 70 \mathrm{ps}$ for $I_{B} \approx 0.98 I_{S W}$. The differences in jitter between the channels can be assumed to be contributed to SNSPD fabrication improvements between device generations. Note that Channels 3 and 4, which are FMF-coupled, had the least jitter indicating that the larger core FMF contributes negligibly. This is in concurrence with theory, from which we estimate a worst case $0.365 \mathrm{ps}$ rms jitter contribution. 


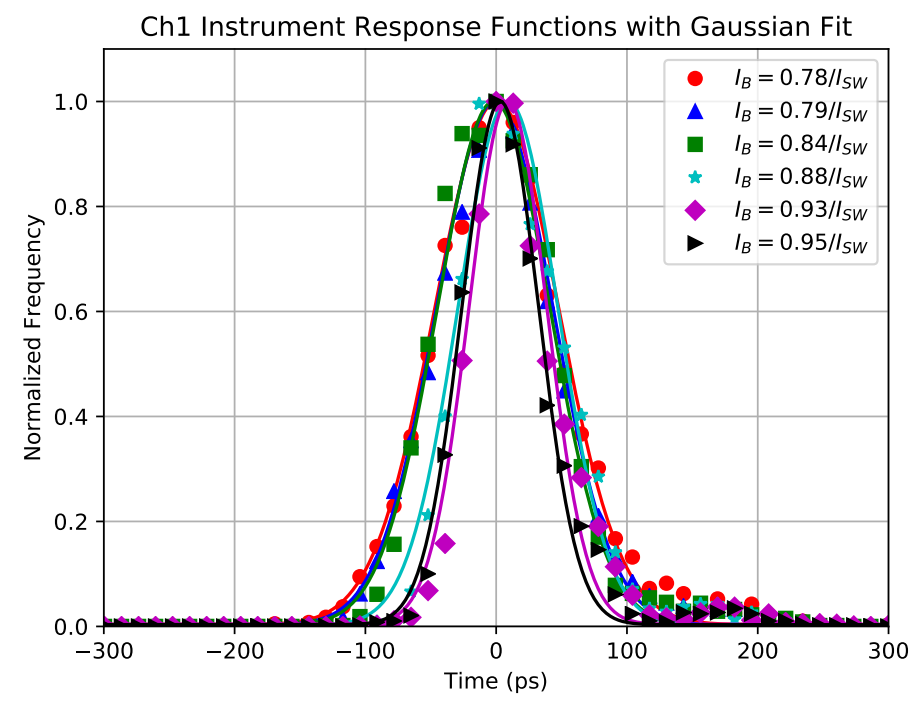

Figure 9: Measured IRFs with Gaussian fits for Channel 1 for different current bias. The measured IRFs for all channels are approximately Gaussian and qualitatively identical.

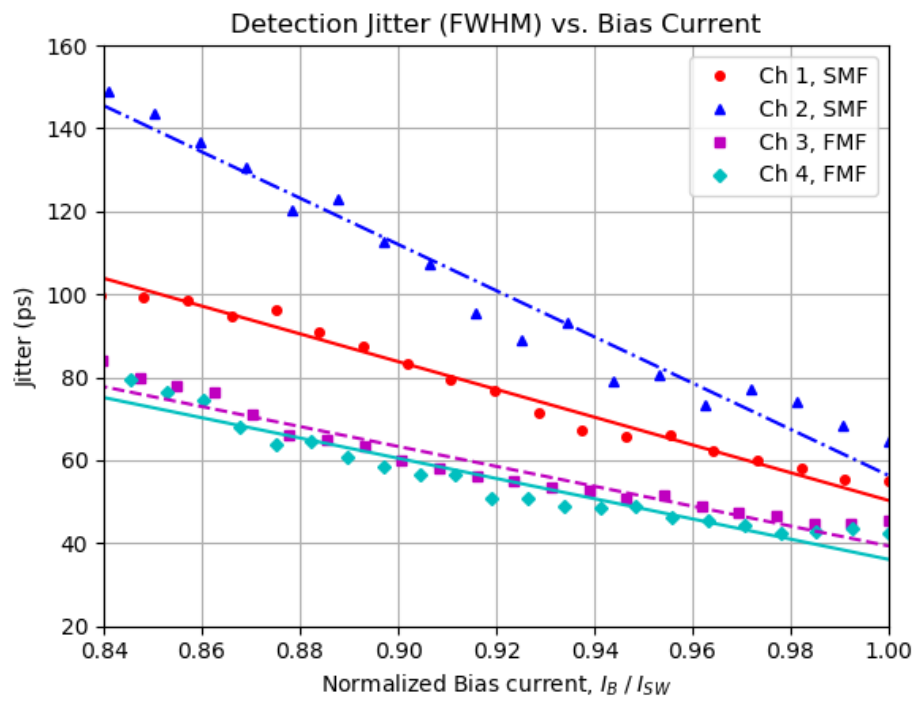

Figure 10: Detector system jitter as a function of bias current.

\section{FEW-MODE FIBER SPATIAL MODE EFFICIENCY}

Of particular interest is the coupling efficiency of the FMF-coupled detectors as the modal content of the input light increases. For applications to free-space optical and quantum optical communications this is important as the atmosphere causes distortions to the spatial profile of the transmitted beam, sending energy into high-order spatial modes. There are options to mitigate this effect including multi-mode fiber (MMF) or free-space coupling to larger area SNSPDs and/or multi-element SNSPD arrays, ${ }^{2}$ MMF coupling to SNSPDs with focusing optics, ${ }^{10}$ and of notable interest MMF to SMF and MMF to FMF photonic lanterns. ${ }^{11}$

A typical MMF to SMF photonic lantern offers a potentially advantageous solution for coupling from a 
telescope to SNSPDs because they allow for the reception of light with higher-order mode content without the need for design and development of large-area SNSPDs or complex cryogenic focusing optics. Large-area SNSPDs tend to have longer dead times and substantially increased jitter and dark count rates compared to typical and commercially available $<\approx 14 \mu \mathrm{m}$ diameter SNSPDs to-date. Thus while advantageous for coupling, can be disadvantageous for high-photon efficiency space-to-ground optical communications applications. However, a distinct disadvantage of the MMF to SMF photonic lantern is in the number of detectors required. In order to detect all of the light collected by a photonic lantern, a separate SMF and therefore SNSPD is required per allowable mode of the MMF input side of the lantern. Since a typical MMF can support 10s to 100s of modes, this could become prohibitively large, especially because in many applications the number of detectors required for the data rate would be less than the lantern requires. The solution to this that we have considered is multi-mode to few-mode fiber photonic lanterns in which the output few-mode fibers are designed to couple as efficiently as single-mode fiber to commercial SNSPDs.

Here we are interested in the detection efficiency of the SNSPDs across the mode spectrum of the FMFs. The FMFs we have considered are 20- $\mu \mathrm{m}$ gradient-index (GRIN) cores with the design profile shown below in Figure 11a. The profile is parabolic with an assumed cladding refractive index of 1.444 and peak index of 1.456.

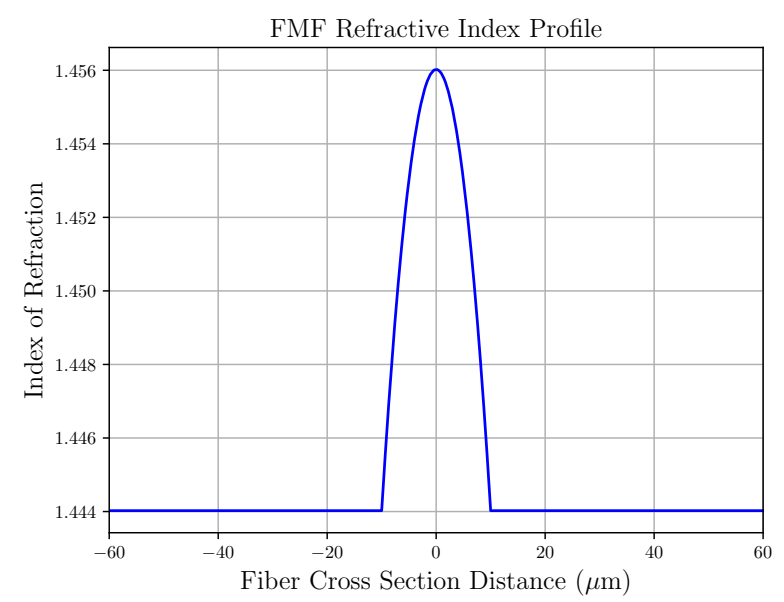

(a)

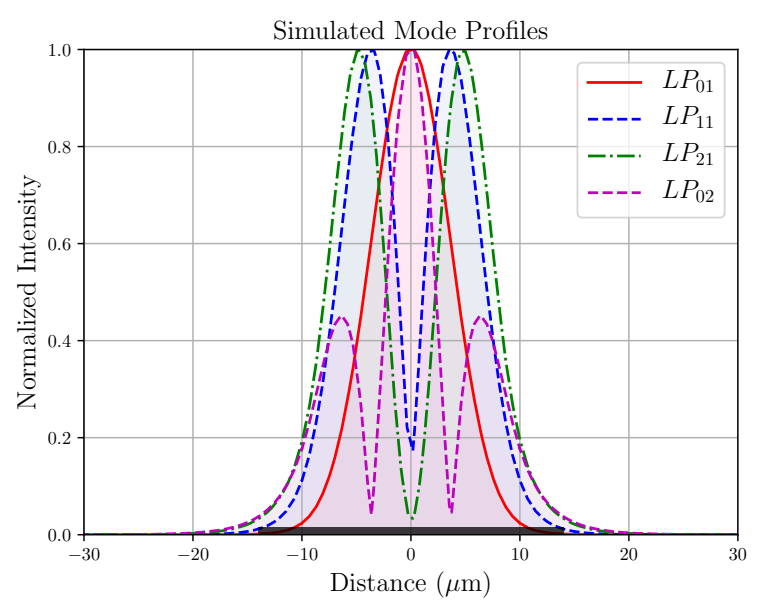

(b)

Figure 11: (a) Few-mode fiber refractive index profile. (b) Calculated mode profiles with 14- $\mu \mathrm{m}$ SNSPD reference diameter.

Not accounting for polarization, this FMF supports up to six $L P$ modes $-L P_{01}, L P_{11 e}, L P_{11 o}, L P_{21 e}, L P_{21 o}$, and $L P_{02}$. Additionally, the GRIN profile of the fiber is such that the mode-field diameter (MFD) of $L P_{01}$ is equal to the MFD for SMF-28, $\approx 10.4 \mu \mathrm{m}$. Shown in Figure $11 \mathrm{~b}$ are the simulated mode profiles from finite-element boundary analysis for each of the FMF modes, omitting degeneracy, with a $14-\mu \mathrm{m}$ line representing the SNSPD diameter for reference. In Table 1 we have the theoretical effective mode area, $A_{\text {eff }}$ for each mode and the corresponding mode-field diameters. The effective mode area was determined from the two-dimensional mode

\begin{tabular}{c|c|c|c|c}
\hline & $L P_{01}$ & $L P_{11}$ & $L P_{21}$ & $L P_{02}$ \\
\hline$A_{\text {eff }}\left(\mu \mathrm{m}^{2}\right)$ & 83.12 & 168.08 & 235.77 & 185.13 \\
\hline $\operatorname{MFD}(\mu \mathrm{m})$ & 10.29 & 14.63 & 17.33 & 15.35 \\
\hline
\end{tabular}

Table 1: Effective mode area and mode field diameter (MFD)

intensity profiles $I(x, y)$ shown in Figure 13a by numerically integrating over the plane using the relation ${ }^{12}$ 


$$
A_{e f f}=\frac{\left[\iint I(x, y) d x d y\right]^{2}}{\iint I^{2}(x, y) d x d y},
$$

and the mode-field diameter was estimated simply through the geometric transform MFD $=(2 / \sqrt{\pi}) \sqrt{A}_{\text {eff }}$.

To measure the mode-dependent system detection efficiency experimentally we used the set-up shown in Figure 12. The main feature of this set-up was a spatial light modulator (SLM), with a $12.8 \times 12.8 \mathrm{~mm}$ surface comprised of an array of $512 \times 512$ active liquid crystal pixels which allowed for the generation of beam profiles of arbitrary spatial shape. To condition the CW $1550 \mathrm{~nm}$ source beam for the SLM we first collimated the source beam using a $4 \mathrm{~mm}$ diameter, $f=15 \mathrm{~mm}$ reflective collimator before being sent through a beam expander to adjust the fill factor on the SLM. Next the beam was passed through a half-wave plate to align the polarization to the SLM's polarization sensitivity axis. The light was then passed through a 50/50 beam splitter with half of the light reaching the SLM, which reflected the now spatially modulated beam back though the 50/50 beam splitter. From the beam splitter we used an $f=18.4 \mathrm{~mm}$ focusing lens to couple light into the input few-mode fiber. Finally we wound the input FMF around a three-paddle fiber polarization controller before inputting into the detector enclosure.

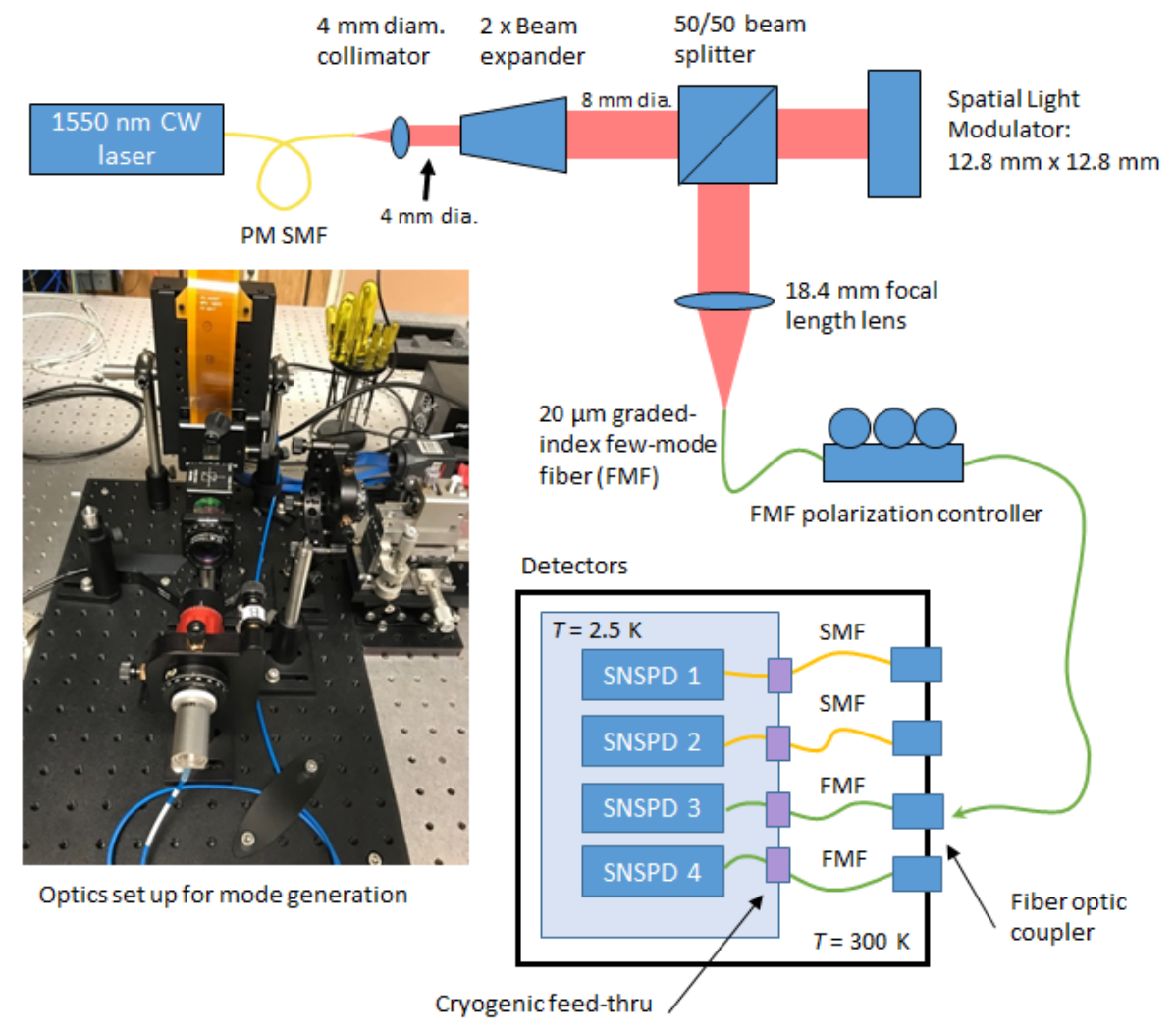

Figure 12: Mode characterization setup.

We generated the $L P_{l m}$ modes shown in Figure 13a using the spatial light modulator by encoding the liquid crystal matrix with the corresponding phase hologram in Figure 13b. Each hologram is the inverse Fourier transform of the desired profile, and when applied to the SLM changes the phase of the input $L P_{01}$ Gaussian beam appropriately. In Figure 13c we show the measured beam intensity directly from the FMF at the detector system input port as measured with a beam profiler. Due to unknown stresses on the FMF between the enclosure input 
and the SNSPD we were not able to verify precisely the shape of the mode incident on the detector. However, by applying a repeatable range of stresses on the input FMF were able to determine in the most extreme cases the correspondence $L P_{01} \leftrightarrow L P_{11}$ and $L P_{21} \leftrightarrow L P_{02}$, with linear linear combinations in between. The detectors
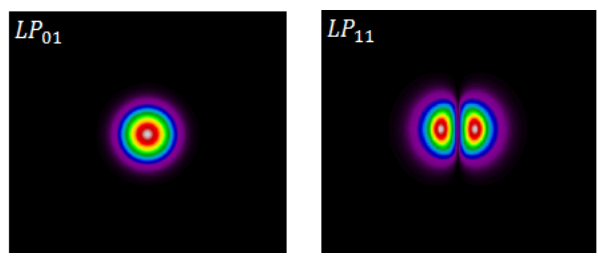

(a)
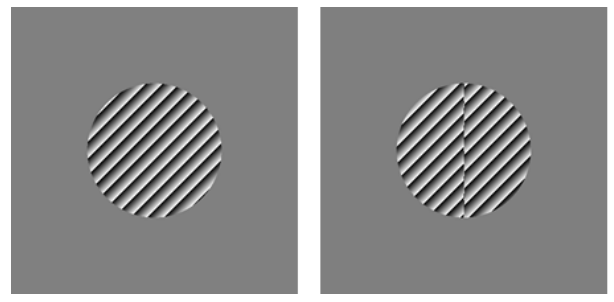

(b)
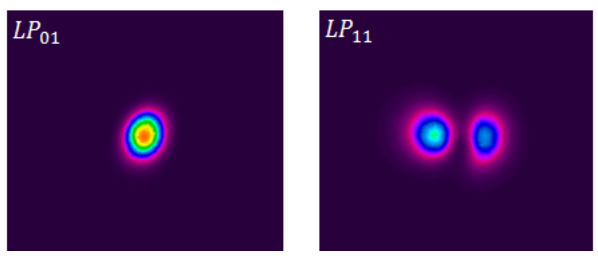
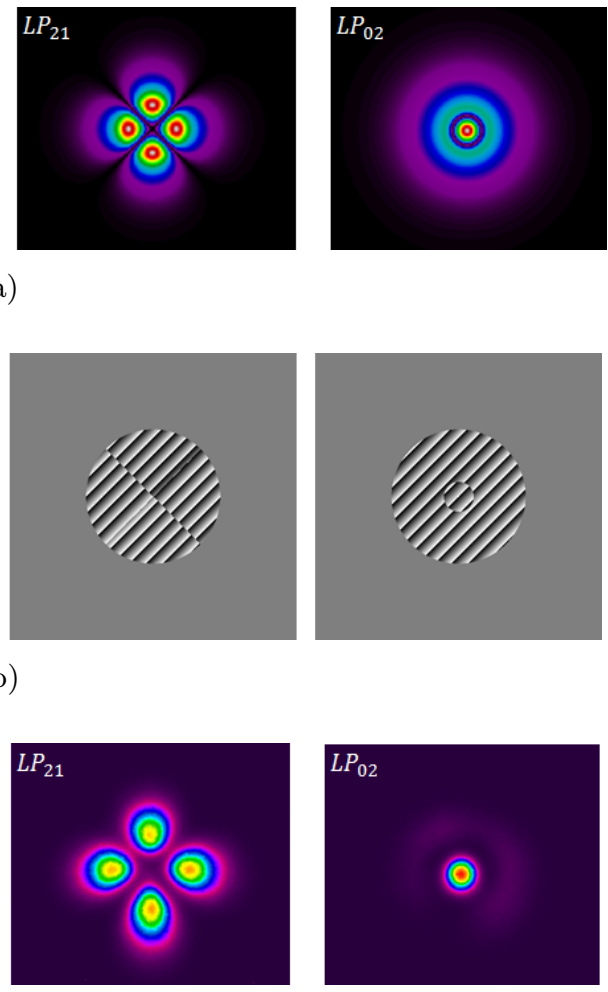

(c)

Figure 13: (a) Theoretical FMF mode profiles. (b) Spatial light modulator phase holograms used to generate the FMF modes. (c) Measured beam profiles at the input to the detector enclosure.

were biased to a level of $I_{B} \approx 0.9 I_{S W}$, and remained constant for all measurements. As before, we attenuated the input photon flux to $R_{i n} \approx 500,000 \mathrm{ph} / \mathrm{s}$ after characterizing the system losses through the SLM optical setup and FMF, and estimated the detection efficiency from $S D E=\left(R_{\text {out }}-B C R\right) / R_{\text {in }}$. All measurements were performed with the room lighting off, and the background count rate $(B C R)$ was measured to be $\approx 35-38$ kcps, similar to what is shown in Figure 3 for the same bias point. The FMF polarization controller (FM-FPC) was adjusted to optimal polarization in each case to obtain maximum output count rate. Since the polarization adjustment is primarily a stress effect this required significant trial-and-error adjustment of the FM-FPC to find an optimal state of polarization and spatial profile, which resulted in a limited set of measurement data, specifically 4 in total for each mode.

\begin{tabular}{l|r|r|r|r}
\hline & $L P_{01}$ & $L P_{11}$ & $L P_{21}$ & $L P_{02}$ \\
\hline$S D E_{\max }(\%)$ & 80.36 & 72.09 & 66.26 & 67.54 \\
\hline$\sigma_{S D E}(\%)$ & 1.03 & 1.76 & 5.13 & 3.51 \\
\hline$S D E_{\text {est }}$ & 80.36 & 76.90 & 64.92 & 73.29 \\
\hline
\end{tabular}

Table 2: Mode-dependent detection efficiency, FMF-coupled detectors.

Figure 14 and Table 2 show the results of these measurements, along with an estimate of the efficiency 
based on the relative detector diameter $d$ to MFD ratio, i.e. $S D E_{\text {est }} \approx S D E_{\max }(d / M F D)$ for $M F D \geq d$ and $S D E_{e s t}=S D E_{\max }$ for $M F D<d$. Here the SNSPD diameter $d$ was assumed to be $14 \mu \mathrm{m}$. These numbers are similar, indicating that the efficiency differences for higher order modes is likely due to detector overfill. The average detection efficiency over all the modes was $71.56 \% \pm 2.86$. For a SMF, we show the efficiency results in Table 3 and also in Figure 14. While for a SMF the detection efficiency for $L P_{l m}$ modes other than $L P_{01}$

\begin{tabular}{l|r|r|r|r}
\hline & $L P_{01}$ & $L P_{11}$ & $L P_{21}$ & $L P_{02}$ \\
\hline$S D E_{\max }(\%)$ & 80.47 & 11.41 & 16.28 & 5.81 \\
\hline$\sigma_{S D E}(\%)$ & 1.19 & 6.08 & 7.14 & 2.78 \\
\hline
\end{tabular}

Table 3: Mode-dependent detection efficiency, SMF-coupled detectors.

should be zero since those modes do not propagate, there was cross-coupling from the high-order modes into $L P_{01}$ introduced at the FMF to SMF coupling joint. When averaged over all the modes, this increased the SMF mode-average detection efficiency from $\approx 20 \%$ to $\approx 28.54$. Compared to the SMF, we can define the average mode gain for the FMF-coupled detectors to be $\approx 2.51$, or about $4.0 \mathrm{~dB}$.

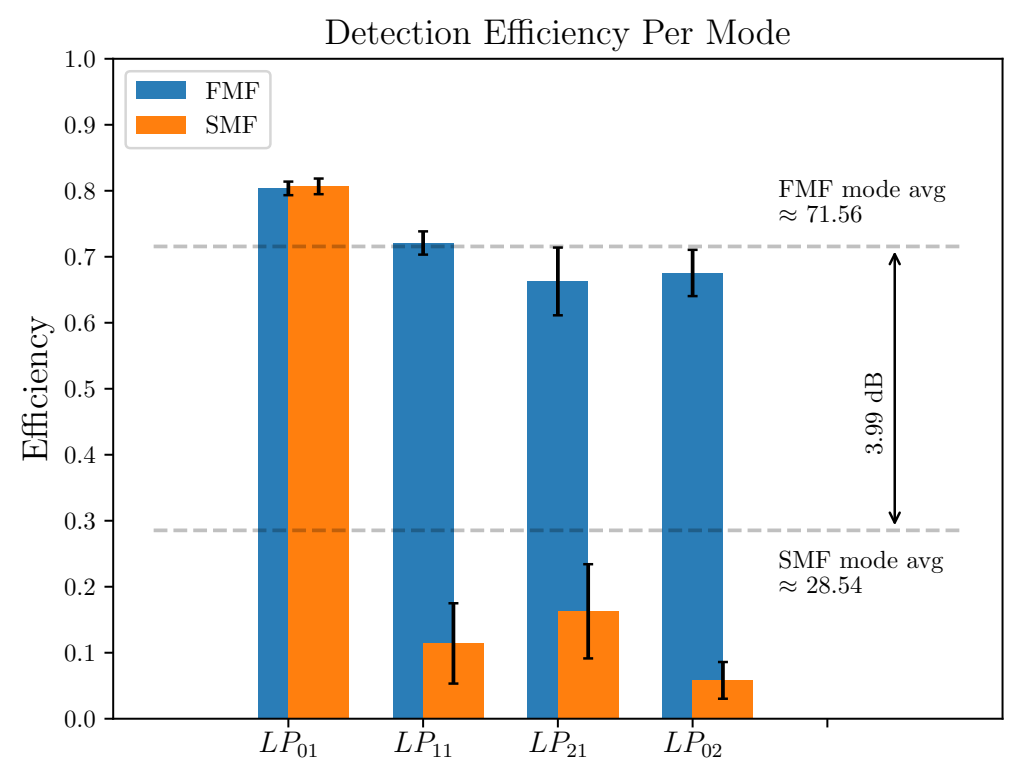

Figure 14: Mode dependent detection efficiency for each individual mode. The dotted lines are the average over all the modes.

\section{PULSE POSITION MODULATION OPTICAL COMMUNICATIONS LINK TESTING RESULTS}

Link testing was performed in our optical communications photon counting test bed, ${ }^{13}$ designed to emulate the transmitter and receiver of photon counting optical communications links. The testbed features an optical software defined radio (SDR) which implements the CCSDS Optical Communications Coding and Synchronization Red Book telemetry link, ${ }^{14}$ and the addition of two series electro-optic high-extinction ratio intensity modulators driven by the SDR. ${ }^{15}$ Previously we demonstrated $20 \mathrm{Mbps}$ error-free performance with a single detector for PPM-32, code rate $1 / 3$, and slot width, $T_{s}=2 \mathrm{~ns},{ }^{3}$ but it was not possible to achieve higher data rates due to the detector blocking time of $\approx 35 \mathrm{~ns}$. With the improvements to our SNSPDs that have reduced the dead time 
to $<25 \mathrm{~ns}$, we selected the same waveform for demonstration but with $T_{s}=1 \mathrm{~ns}$ for a $40 \mathrm{Mbps}$ data rate. For the purposes of this testing we used the SNSPDs coupled to SMF (Channels 1 and 2) since this was our previous baseline. Figure 15 shows the bit error rate (BER) testing results along with simulated curves for very low noise conditions $\left(K_{b} \approx 0.0001\right)$. The simulations assumed a detector dead time of $20 \mathrm{~ns}$, and total RMS system jitter of $61 \mathrm{ps}$ and $68 \mathrm{ps}$. We were able to close the $40 \mathrm{Mbps}$ link error-free, and the simulations matched very closely with the measured results.

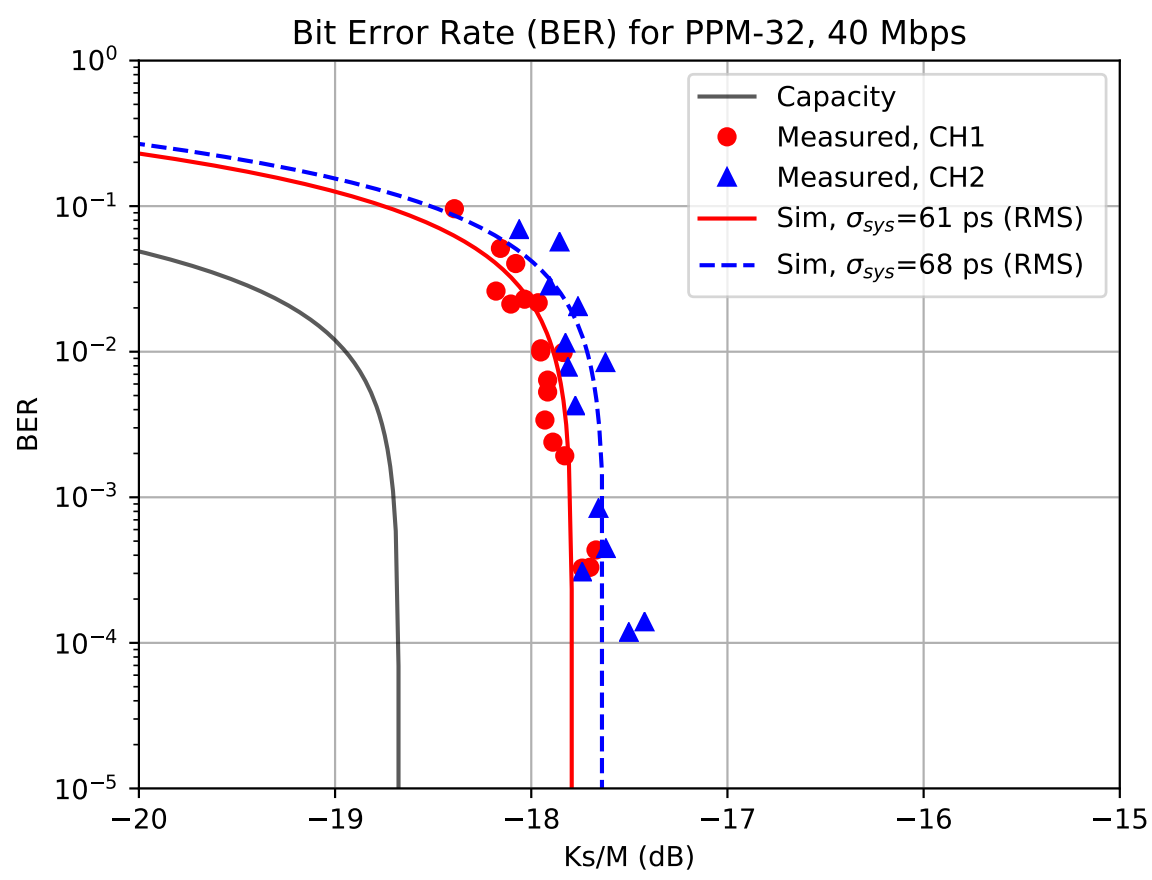

Figure 15: Optical link testing BER curve results for PPM-32, 40 Mbps waveform with $K_{b} \approx 0.0001$. The simulation curves are for two different total system jitter, and an assumed $20 \mathrm{~ns}$ detector dead time.

\section{CONCLUSION}

We characterized a commercial superconductor nanowire single-photon detector system with improved detector count rates, and explored coupling to few-mode fibers (FMF). Parameters such as detection efficiency, dark count rate, reset time, maximum count rate, and timing jitter were assessed, along with detection efficiency for the higher-order fiber modes supported by the FMFs, $L P_{01}, L P_{11}, L P_{21}$, and $L P_{02}$. Detector dead time was reduced to less than $25 \mathrm{~ns}$, improving the overall count rates to over $60 \mathrm{Mcps}$ per channel, and jitter was shown to be less than $80 \mathrm{ps}(\mathrm{FWHM})$ in the detector operating region, approaching $\approx 45 \mathrm{ps}(\mathrm{FWHM})$ in the best case. When averaged across the FMF modes, the FMF-coupled detectors had nearly $5.5 \mathrm{~dB}$ detection efficiency gain as compared to SMF. With a single detector a $40 \mathrm{Mbps}$ communications link was closed error-free, and the BER results validated our simulation model. Future work will include communication link performance improvement through combining multiple detector channels to achieve higher rates, multi-mode to single-mode and multimode to few-mode fiber photonic lantern characterization, and link performance with emulated atmospheric turbulence.

\section{ACKNOWLEDGMENTS}

The authors would like to acknowledge the support by the NASA Space Communications and Navigation (SCaN) program, and Aaron Miller and Tim Rambo from Quantum Opus LLC for useful discussions. 


\section{REFERENCES}

[1] Robinson, B. S., Boroson, D. M., Burianek, D. A., Murphy, D. V., Khatri, F. I., Burnside, J. W., Kansky, J. E., Biswas, A., Sodnik, Z., and Cornwell, D. M., "The nasa lunar laser communication demonstration successful high-rate laser communications to and from the moon," in [AIAA - SpaceOps 2014], (2014).

[2] Shaw, M. D., Marsili, F., Beyer, A. D., Stern, J. A., Resta, G. V., Ravindran, P., Chang, S., Bardin, J., Russell, D. S., Gin, J. W., Patawaran, F. D., Verma, V. B., Mirin, R. P., Nam, S. W., and Farr, W. H., "Arrays of WSi superconducting nanowire single photon detectors for deep-space optical communications," in [CLEO: 2015], OSA Technical Digest (online) (Optical Society of America, 2015), paper JTh2A.68.

[3] Vyhnalek, B. E., Tedder, S. A., and Nappier, J. M., "Performance and characterization of a modular superconducting nanowire single photon detector system for space-to-earth optical communications links," in [Proc. SPIE 10524 - Free-Space Laser Communication and Atmospheric Propagation XXX], (2018).

[4] Quantum Opus, Nanowire Datasheet (Jan. 2017).

[5] Miller, A. J., Lita, A. E., Calkins, B., Vayshenker, I., Gruber, S. M., and Nam, S. W., "Compact cryogenic self-aligning fiber-to-detector coupling with losses below one percent," Optics Express 19, 9102 - 9110 (May 2011).

[6] Quantum Opus, Nanowire Bias and Readout Electronics Datasheet (Jan. 2017).

[7] Schätzel, K., Kalström, R., Stampa, B., and Ahrens, J., "Correction of detection-system dead-time effects on photon-correlation functions," J. Opt. Soc. Am. B 6, 937-947 (May 1989).

[8] Marsili, F., Verma, V. B., Stern, J. A., Harrington, S., Lita, A. E., Gerrits, T., Vayshenker, I., Baek, B., Shaw, M. D., Mirin, R. P., and Nam, S. W., "Detecting single infrared photons with $93 \%$ system efficiency," Nature Photonics 7, 210 - 214 (2013).

[9] Verma, V. B., Korzh, B., Bussières, F., Horansky, R. D., Dyer, S., Lita, A., Vayshenker, L., Marsili, F., Shaw, M. D., Zbinden, H., Mirin, R. P., and Nam, S. W., "High-efficiency superconducting nanowire single-photon detectors fabricated from MoSi thin-films," Optics Express 23 (Dec. 2015).

[10] Grein, M. E., Kerman, A. J., Dauler, E. A., Shatrovoy, O., Molnar, R. J., Rosenberg, D., Yoon, J., DeVoe, C. E., Murphy, D. V., Robinson, B. S., and Boroson, D. M., "Design of a ground-based optical receiver for the lunar laser communications demonstration," in [2011 International Conference on Space Optical Systems and Applications (ICSOS)], (2011).

[11] Tedder, S. A., Vyhnalek, B. E., Leon-Saval, S., Betters, C., Floyd, B., Staffa, J., and Lafon, R., "Singlemode fiber and few-mode fiber photonic lanter performance evaluated for use in a scalable real-time photon counting ground receiver," in [Proc. SPIE 10910 - Free-Space Laser Communication and Atmospheric Propagation XXXI], (2019).

[12] Agrawal, G., [Nonlinear Fiber Optics], Academic Press, 2 ed. (1995).

[13] Nappier, J. M., Vyhnalek, B. E., Tedder, S. A., and Lantz, N. C., "Characterization of a photon counting test bed for space to ground optical pulse position modulation communications links," in [Proc. SPIE 10910 - Free-Space Laser Communication and Atmospheric Propagation XXXI], (2019).

[14] Consultative Committee for Space Data Systems (CCSDS), Optical Communications Coding and Synchronization Draft Recommended Standard (2018).

[15] Lantz, N. C., Nappier, J. M., Vyhnalek, B. E., and Tedder, S. A., "Optical software defined radio transmitter extinction ratio enhancement with differential pulse carving," in [Proc. SPIE 10910 - Free-Space Laser Communication and Atmospheric Propagation XXXI], (2019). 